\title{
LAITS HUMANISÉS
}

\section{par \\ José Maria ROSELL et JUAN MINUT}

Nous avons le plaisir de présenter, en deux articles, dont le premier ci-après, la substance d'un chapitre du très bel ouvrage (1) qui vient de parâ̂tre et qui représente, en deux forts volumes, avec une abondante illustration, un document de grande valeur.

En raison de leur intérêt actuel, les deux textes dont il s'agit ont fait l'objet d'une publication préalable, sensiblement analogue, dans la très estimée Revue "La Industria. Lechera-Republique Argentine " au cours de l'année écoulée.

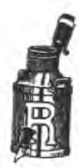

Etant donné la grande importance de cette branche de la lactologie, nous jugeons tout indiqué de présenter une révision des conceptions modernes et des progrès réalisés dans ces types de laits.

En ce qui concerne les laits maternisés, mieux encore : humanisés, ou ainsi dénommés, qui se préparent et même se fabriquent jusqu'à maintenant, on applique le principe, courant depuis plusieurs dizaines d'années, de diluer les laits avec de l'eau ou avec quelque décoction de céréales. Par cette dilution, on rapproche la teneur en caséine du lait de vache de celle du lait humain, et on ajoute en outre divers sucres au lait, car le lactose s'observe en plus grande quantité dans le lait humain. Bien que, pour des motifs de commodité ou à cause de difficultés d'exécution, on continue d'utiliser ces types de lait avec plus ou moins d'extension, les pédiatres de la plupart des pays réclament des laits maternisés sur des bases plus physiologiques.

Au cours de ces dernières années, les études approfondies et les nombreuses expériences cliniques de grands pédiatres tels que Czerny, Keller, Ugnade, Arnold, Musher, Percival, Nichelson, Finkelstein, Müller, Gonce, Marriot, Davidson, Hesen, Mazner, Kerley, Brenne, Mam, Bessau, Marfan, Spolverini, etc., ont démontré l'insuffisance des principes primaires et la nécessité de considérer plusieurs autres aspects qui différencient le lait de vache du lait humain.

Les principales différences reconnues depuis longtemps reposent sur les teneurs du lait en sucre, caséine, lactoalbumine et sels, différences qui ressortent clairement lorsqu'on examine les

(1) Méthodes analytiques de Laboratoire Lactologique et Microbiologie des Indus, tries laitières (en langue espagnole), par $D^{r}$ José $M$. Rosell et $D^{r}$ Inacio Dos SantosEditeur : Editorial Labor S. A,, Barcelone, Madrid, Buenos-Aires, Rio-de-Janeiro. Mexico, Montevideo, 1952. 
chiffres des analyses effectuées par de nombreux chercheurs. D'après les chiffres de KoEnig (confirmés par l'un de nous, après des centaines d'analyses, comme étant égaux en Espagne), le lait de vache, comparé au lait maternel humain, comprend en moyenne:

$\begin{array}{cr}\text { Lait de vache } & \text { Lait de femm } \\ 1,0313 & 1,0298 \\ 87,07 \% & 87,58 \% \\ 3,02 \% & 0,70 \% \\ 0,51 \% & 1,21 \% \\ 3,08 \% & 3,84 \% \\ 4,94 \% & 6,37 \% \\ 0,72 \% & 0,30 \%\end{array}$

\section{Les vitamines}

La teneur en vitamines varie beaucoup dans le colostrum et le lait de vache, dans le colostrum et le lait humain. Dans les colostrums, cette teneur décroît lors du passage de la sécrétion colostrale à la lactation normale.

Par exemple, à Buenos-Aires, les docteurs Escudero et PieranGELI ("Influence de l'alimentation sur la richesse minérale et en vitamines du colostrum ", Diétologie, vol. I, 1943), trouvèrent ces chiffres moyens dans la composition vitaminée de 101 colostrums du deuxième jour :

\begin{tabular}{|c|c|c|}
\hline Provitamine A & 316,9 & U.I. \\
\hline Vitamine A préformée $\ldots . . .$. & 170,3 & ") \\
\hline otal vitamine $\mathrm{A} \ldots \ldots \ldots \ldots \ldots \ldots \ldots \ldots \ldots$ & 487,2 & $n$ \\
\hline 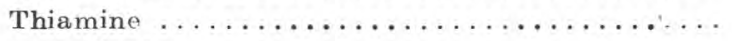 & 10,2 & mmas \\
\hline 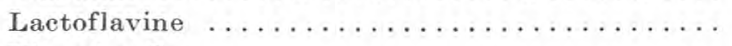 & 36,5 & n \\
\hline itamine $\mathrm{C} \ldots \ldots \ldots \ldots$ & 2,30 & mg. \\
\hline
\end{tabular}

Les doctoresses Herrais et Herrero ont mis en lumière de grandes variations des vitamines dans le colostrum des accouchées, à la maternité de l'hôpital Durand à Buenos-Aires :

\begin{tabular}{|c|c|c|c|c|c|c|c|}
\hline $\begin{array}{c}\text { Jour après } \\
\text { l'accou- } \\
\text { ehement, } \\
\text { quand fut } \\
\text { pris l'é- } \\
\text { chantillon }\end{array}$ & $\begin{array}{c}\text { Provita- } \\
\text { mine A } \\
\text { (U. I. } \\
\text { par litre) }\end{array}$ & $\begin{array}{c}\text { Vitamine } \\
\text { A } \\
\text { préformée } \\
\text { (U. I. } \\
\text { par litre) }\end{array}$ & $\begin{array}{c}\text { Provita- } \\
\text { mine }+ \\
\text { vitamine } \\
\text { A } \\
\text { préformée }\end{array}$ & $\begin{array}{l}\text { Vitamine } \\
\text { C. (mgr. } \\
\text { par litre) }\end{array}$ & $\begin{array}{c}\text { Tiamine } \\
\text { (gammas } \\
\text { par litre) }\end{array}$ & $\begin{array}{c}\text { Lactofla- } \\
\text { vine } \\
\text { (gammas } \\
\text { par litre) }\end{array}$ & $\begin{array}{c}\text { Acide } \\
\text { nicotini- } \\
\text { que } \\
\text { (mgr. par } \\
\text { litre) }\end{array}$ \\
\hline$\ldots \ldots \ldots$ & 9,333 & 9,493 & 10,826 & 35 & 125 & 250 & 1 \\
\hline$\ldots \ldots$ & 1,598 & 1,395 & 2,993 & 23 & 84 & $\begin{array}{c}430 \\
\text { page } 97,\end{array}$ & $\begin{array}{r}1 \\
1943 .)\end{array}$ \\
\hline
\end{tabular}


Dans le colostrum de vache, la teneur en vitamine varie considérablement. Nous avons extrait les données les plus dignes d'attention, concernant les premiers jours de la parturition, et nous les résumons comme suit :

Vitamine A, 5 à 15 fois plus que le lait.

Vitamine B1, 10 fois plus que le lait.

Vitamine B2, teneur élevée.

Vitamine C, riche.

Vitamines E, K, P, P, plus ou moins, comme le lait.

Quant aux vitamines les plus connues contenues dans le lait humain et le lait de vache, les valeurs suivantes sont considérées comme normales :

\begin{tabular}{ll|c|c}
\hline \multicolumn{2}{c|}{ Vitamines dans $100 \mathrm{~cm}^{3}$} & Lait de vache & Lait humain \\
\cline { 3 - 4 } & & & \\
A & U.I. & $50-600$ & $250-400$ \\
B1 & mg. & $20-75$ & $50-60$ gammas \\
B2 & mg. & $100-300$ & $10-25$ gammas \\
PP & mg. & 0,11 & $?$ \\
C & mg. & $0,3-3,9$ & 4,20 \\
D & U.I. & $0,5-10$ & 0,80 \\
E & mg. & 0,02 & $?$ \\
Pyridoxine & mg. & $0,048-0,067$ & $?$ \\
Inositol & microgrammes & 18 & $?$ \\
\hline \hline
\end{tabular}

D'accord avec l'observation objective du dosage des vitamines dans les deux laits, il y a lieu de reconnaitre avant tout que la teneur la plus stable ou normalisée (sauf variations saisonnières) se rencontre dans le lait de vache. Les vitamines varient amplement dans le lait humain, et l'on considère comme normaux ces résultats, dans 10 millilitres:

\begin{tabular}{|c|c|c|}
\hline Provitamine A $\ldots \ldots \ldots$ & U.I. & $140-160$ \\
\hline Vitamine A préformée . . . . . . . . & ” & 100 \\
\hline Provitamine plus vit. A préformée.. & ) & 300 \\
\hline Vitamine $\mathrm{C} \ldots \ldots \ldots \ldots \ldots \ldots$ & mg. & 0,4 \\
\hline Thiamine $\ldots \ldots \ldots \ldots \ldots \ldots$ & gammas & 25 \\
\hline Lactoflavine . . . . . . . . . . . . & , & 20 \\
\hline
\end{tabular}

\section{Les bactéries}

Sans crainte de nous tromper, nous pourrions affirmer que le lait humain acheté ou cédé dans des "lactariums" (pouponnières, etc.) ou dans le privé, doit être contrôlé comme le lait de vache. Le choix de la donneuse, la technique de la récolte, reçue directement 
dans des flacons calibrés, les mesures, la stérilisation du matériel, les températures à observer pour sa bonne conservation, etc., sont des pratiques qui conduisent à la "certification " du lait humain. Sans elles, le nourrisson est exposé aux infections, aux troubles de la nutrition et aux maladies. Pour pallier ces inconvénients, on a adopté la pasteurisation, l'ébullition, etc.

WEISMAN et SAVIANo ont trouvé, dans quarante laits humains en circulation dans la ville de Buenos-Aires, un minimum de 540 germes par millilitre et un maximum de 325.000. La moyenne arithmétique générale se rapproche de 100.000 bactéries par centimètre cube.

Ils décelèrent dans onze laits la présence du "colibacille ", dans une proportion évaluée à $27 \%$ des laits examinés.

Si nous prenons en considération les conclusions des auteurs précités, il apparaît :

a) Que $80 \%$ des laits des lactariums sont peuplés de bactéries à l'excès, et

b) Que $60 \%$ des laits de même origine sont contaminés par le "colibacille »; nous devons convenir de la nécessité d'un rigoureux contrôle bactériologique réglementant la commercialisation ou l'utilisation de ces laits.

Il faut aussi rechercher les tromperies possibles, condamnables, par addition de lait de vache. Tant l'obtention de ces laits que leur inspection complète, leur modification, etc., doivent être confiées à un laboratoire lactologique de capacité reconnue.

\section{Le colostrum}

Les composants des colostrums (humain et de vache) sont très variables et, en particulier, les études faites sur le colostrum humain ont démontré l'influence marquée de l'alimentation de l'accouchée.

Les différences trouvées à Buenos-Aires étaient :

\begin{tabular}{|c|c|c|}
\hline Par litre & $\begin{array}{c}\text { Colostrum } \\
\text { humain }\end{array}$ & $\begin{array}{l}\text { Colostrum } \\
\text { de vache }\end{array}$ \\
\hline Hydrates de carbone & 51,80 & 29,60 \\
\hline Protéines $\ldots \ldots \ldots \ldots \ldots \ldots \ldots \ldots$ & 21,20 & 106,90 \\
\hline Matières grasses $\ldots \ldots \ldots \ldots \ldots \ldots \ldots$ & $21,5 \oplus$ & 42,50 \\
\hline
\end{tabular}

La valeur totale en calories est très différente, à savoir :

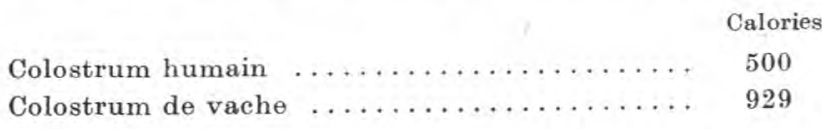

Les matières minérales se différencient de la manière suivante: 


\begin{tabular}{|c|c|c|c|c|}
\hline & \multicolumn{2}{|c|}{ Colostrum humain } & \multicolumn{2}{|c|}{ Colostrum de vache } \\
\hline & $\operatorname{mgr} . \%$ & Pourcentage & mgr. $\%$ & Pourcentage \\
\hline Cendres $\ldots \ldots \ldots \ldots \ldots$. & 3.470 & & 9,200 & \\
\hline Chlore $\ldots \ldots \ldots \ldots \ldots \ldots$ & 850 & 24,49 & 1,040 & 11,30 \\
\hline Potassium $\ldots \ldots \ldots \ldots \ldots$ & 730 & 21,03 & 1,456 & 15,82 \\
\hline Sodium ............. & 460 & 13,25 & 563 & 6,11 \\
\hline Calcium $\ldots \ldots \ldots \ldots \ldots$ & 280 & 8,06 & 1,680 & 18,26 \\
\hline Soufre $\ldots \ldots \ldots \ldots \ldots \ldots$ & 156 & 4,41 & - & - \\
\hline Phosphore ........... & 140 & 4,03 & 1,490 & 16,19 \\
\hline Magnésium $\ldots \ldots \ldots \ldots \ldots$ & 30 & 0,86 & 160 & 1,73 \\
\hline Fer $\ldots \ldots \ldots \ldots \ldots \ldots$ & 1 & 0,02 & - & - \\
\hline Cuivre $\ldots \ldots \ldots \ldots \ldots$ & 0,46 & 0,01 & - & - \\
\hline
\end{tabular}

Ceci sans compter les quelques milligrammes de zinc, d'iode, de plomb, etc., que l'on trouve dans le lait de vache, mais non dans le lait de femme.

D'autres chercheurs ont mis en lumière des traces de vanadium, d'étain, de mercure, etc., attribuant à chacun de ces éléments quelque fonction ou importance.

C'est cette autre raison pour laquelle le médecin, le pédiâtre et le technicien qui préparent les aliments destinés aux prématurés, aux nouveau-nés et aux enfants ont ces indications toujours présentes.

La teneur du colostrum hủmain en éléments minéraux est fonction de l'alimentation de l'accouché. Les différences qui s'observent entre les divers colostrums, en calcium, fer et vitamines quelconques doivent être prises en considération et ont leur importance alimentaire sur le nouveau-né. Tel lait qui est légèrement acide ou neutre quand il est récemment tiré, est habituellement acide lorsqu'on le consomme. Le lait humain est neutre ou alcalin.

On rencontre cinq à six fois plus de caséine dans le lait de vache que dans le lait humain. La raison en est bien simple si nous nous souvenons que le rapport de croissance entre le veau et l'enfant est approximativement de $2: 1$. En outre, la protéine du lait de vache se compose principalement de caséine $(3,02 \%)$ et d'un peu de lactoalbumine $(0,53 \%)$ alors que le lait humain contient de 0,50 à $0,75 \%$ de caséine et $1,23 \%$ de lactoalbumine.

Ceci dit, si nous examinons et comparons les acides aminés, les dissemblances apparaissent de nouveau.

Etant donné que le « colostrum humain et le colostrum de vache représentent un aliment irremplaçable - c'est nous qui le disons ayant une action spécifique et paraspécifique bien déterminée pendant la courte période qui suit la naissance " (les auteurs cités), il 
serait intéressant de modifier et de rapprocher le colostrum de vache du colostrum humain, quand celui-ci manque.

Le lait de vache est plus opaque que le lait humain, bien que ce dernier contienne un plus grand pourcentage de matière grasse. Ceci est dû à l'opacité du caséinate calcique, qui se trouve en plus grande porportion dans le lait de vache.

\section{Les acides aminés}

Examinons les intéressants tableaux ci-après donnant les acides aminés des mêmes protéines dans les laits de vache et de femme.

GOMPOSITION QUANTITATIVE GOMPARÉE DES DIFFÉRENTS ACIDES AMINÉS ENTRE LA GASÉINE, LA LAGTOALBUMINE DU LAIT DE VACHE ET DU LAIT HUMAIN (SELON WILLIAMSON)

\begin{tabular}{|c|c|c|c|c|}
\hline & \multicolumn{2}{|c|}{ Lait de vache } & \multicolumn{2}{|c|}{ Lait humain } \\
\hline & caséine \% & albumine $\%$ & caséine \% & albumine $\%$ \\
\hline$\cdot$ & & & & \\
\hline Valine .............. & 5,3 & 4,0 & 5,0 & 4,1 \\
\hline Leucine . . . . . . . . . & 14,4 & 17,4 & 12,2 & 16,7 \\
\hline Isoleucine . . . . . . . . . & 5,2 & 4,2 & 6,3 & 4,3 \\
\hline Phénylalamine $\ldots \ldots \ldots \ldots$ & 5,5 & 4,5 & 5,8 & 4,8 \\
\hline Méthionine $\ldots \ldots \ldots \ldots \ldots$ & 3,1 & 2,4 & 2,3 & 1,7 \\
\hline Thréonine ............. & 4,6 & 4,3 & 4,5 & 4,0 \\
\hline Lysine $\ldots \ldots \ldots \ldots \ldots$ & 6,0 & 6,2 & 5,6 & 6,6 \\
\hline Arginine $\ldots \ldots \ldots \ldots \ldots$ & 3,9 & 3,6 & 3,4 & 5,0 \\
\hline Hystidine .............. & 2,0 & 1,4 & 2,0 & 1,5 \\
\hline Tryptophane ........... & 1,3 & 2,1 & 1,5 & 2,3 \\
\hline Glycocolle............. & 0,4 & 0,0 & 0,0 & 0,0 \\
\hline Alanine ................ & 2,3 & 2,6 & 2,0 & 2,5 \\
\hline Tyrosine $\ldots \ldots \ldots \ldots \ldots$ & 5,5 . & 3,5 & 5,5 & 4,5 \\
\hline Sérine $\ldots \ldots \ldots \ldots \ldots \ldots$ & 5,0 & 4,0 & 5,4 & 4,2 \\
\hline Cystine $\ldots \ldots \ldots \ldots \ldots \ldots$ & 0,4 & 3,1 & 0,6 & 3,8 \\
\hline Asparagine ............ & 4,2 & 9,6 & 4,6 & 9,3 \\
\hline Glutamine $\ldots \ldots \ldots \ldots$ & 21,9 & 13,7 & 20,9 & 12,5 \\
\hline Proline $\ldots \ldots \ldots \ldots \ldots \ldots$ & 8,1 & 4,0 & 8,9 & 3,5 \\
\hline
\end{tabular}

POURGENTAGE TOTAL DES DIFFÉRENTS AGIDES AMINÉs POUR L'ENSEMBLE DU LAIT

\begin{tabular}{|c|c|c|}
\hline & $\begin{array}{l}\text { Lait de vache } \\
\qquad \% \\
2,8 \text { caséine } \\
0,5 \text { albumine }\end{array}$ & $\begin{array}{l}\text { Lait humain } \\
\% \\
0,5 \text { caséine } \\
1,0 \text { albumine }\end{array}$ \\
\hline Valine $\ldots \ldots \ldots \ldots \ldots \ldots$ & 0,171 & 0,066 \\
\hline Leucine . . . . . . . . . . . . & 0,490 & 0,288 \\
\hline Isoleucine $\ldots \ldots \ldots \ldots \ldots \ldots$ & 0,167 & 0,075 \\
\hline Phénylalamine ........... & 0,177 & 0,077 \\
\hline
\end{tabular}




\begin{tabular}{|c|c|c|}
\hline Méthionine $\ldots \ldots \ldots \ldots \ldots \ldots$ & 0,099 & 0,029 \\
\hline Thréonine ............ & 0,151 & 0,063 \\
\hline Lysine $\ldots \ldots \ldots \ldots \ldots \ldots$ & 0,200 & 0,094 \\
\hline Arginine $\ldots \ldots \ldots \ldots \ldots$ & 0,127 & 0,067 \\
\hline Hystidine $\ldots \ldots \ldots \ldots \ldots \ldots$ & 0,063 & 0,025 \\
\hline Tryptophane ........... & 0,047 & 0,031 \\
\hline Glycocolle .............. & 0,011 & 0,000 \\
\hline Alanine................. & 0,075 & 0,035 \\
\hline Tyrosine $\ldots \ldots \ldots \ldots \ldots \ldots$ & 0,172 & 0,073 \\
\hline Sérine $\ldots \ldots \ldots \ldots \ldots \ldots$ & 0,160 & 0,069 \\
\hline Cystine................. & 0,027 & 0,041 \\
\hline Asparagine $\ldots \ldots \ldots \ldots \ldots \ldots$ & 0,166 & 0,116 \\
\hline Glutamine $\ldots \ldots \ldots \ldots \ldots$ & 0,680 & 0,230 \\
\hline Proline $\ldots \ldots \ldots \ldots \ldots \ldots \ldots$ & 0,250 & 0,080 \\
\hline
\end{tabular}

TABLEAU DES AGIDES AMINÉS DES LAITS DE VAGHe DILUÉS, LAIT LACTOALBUMINE ET LAIT HUMAIN

\begin{tabular}{|c|c|c|c|c|c|}
\hline & Lait $(2 / 5)$ & Lait $(1 / 2)$ & Lait $(2 / 3)$ & $\begin{array}{c}\text { Lait } \\
\text { (lactoalb.) }\end{array}$ & $\begin{array}{l}\text { Lait } \\
\text { humain }\end{array}$ \\
\hline \multicolumn{6}{|l|}{ Acides aminés essentiels } \\
\hline Valine $\ldots \ldots \ldots$ & 0,069 & 0,086 & 0,114 & 0,081 & 0,066 \\
\hline Leucine......... & 0,196 & 0,245 & 0,325 & 0,301 & 0,228 \\
\hline Isoleucine $\ldots \ldots$ & 0,067 & 0,083 & 0,110 & 0,083 & 0,075 \\
\hline Phénylalamine ... & 0,071 & 0,088 & 0,117 & 0,088 & 0,077 \\
\hline Méthionine ..... & 0,040 & 0,050 & 0,067 & 0,048 & 0,029 \\
\hline Thréonine ...... & 0,061 & 0,076 & 0,101 & 0,081 & 0,063 \\
\hline Lysine......... & 0,080 & 0,100 & 0,133 & 0,112 & 0,094 \\
\hline Arginine $\ldots \ldots \ldots$ & 0,052 & 0,064 & 0,085 & 0067 & 0067 \\
\hline Hystidine ...... & 0,025 & 0,032 & 0,042 & 0,029 & 0,025 \\
\hline Tryptophane .... & 0,019 & 0,024 & 0,032 & 0,034 & 0,031 \\
\hline \multirow{2}{*}{\multicolumn{6}{|c|}{$\begin{array}{l}\text { Acides aminés non } \\
\text { essentiels }\end{array}$}} \\
\hline & & & & & \\
\hline Glycocolle ..... & 0,005 & 0,006 & 0,008 & 0,002 & 0,000 \\
\hline Alanine $\ldots \ldots \ldots$ & 0,029 & 0,037 & 0,049 & 0,046 & 0,035 \\
\hline Tyrosine ...... & 0,069 & 0,086 & 0,115 & 0,076 & 0,073 \\
\hline Sérine ......... & 0,068 & 0,080 & 0,106 & 0,079 & 0,069 \\
\hline Cystine $\ldots \ldots \ldots$ & 0,011 & 0,014 & 0,019 & 0,041 & 0,041 \\
\hline Asparagine ..... & 0,066 & 0,083 & 0,110 & 0,143 & 0,116 \\
\hline Glutamine ...... & 0,262 & 0,340 & 0,450 & 0,301 & 0,320 \\
\hline Proline $\ldots \ldots \ldots$ & 0,100 & 0,125 & 0,168 & 0,698 & 0,080 \\
\hline Albumine totale $\% \ldots$ & 1,3 & 1,7 & 2,2 & 1,8 & 1,5 \\
\hline
\end{tabular}

Le lait humain est plus riche en tryptophane, en arginine, 
cystine et autres acides aminés, mais il est moins riche en autres éléments.

Le sucre, dans les deux laits, diffère beaucoup en quantité, mais non en nature. Le lait de vache contient plus du triple de sels organiques que le lait humain.

Les enfants que l'on alimente avec du lait entier de vache vivent, par conséquent, sur un plan plus élevé et différent de métabolisme minéral que ceux que l'on alimente avec le lait maternel.

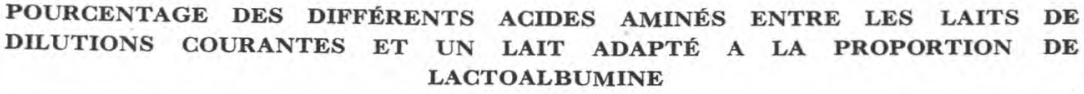

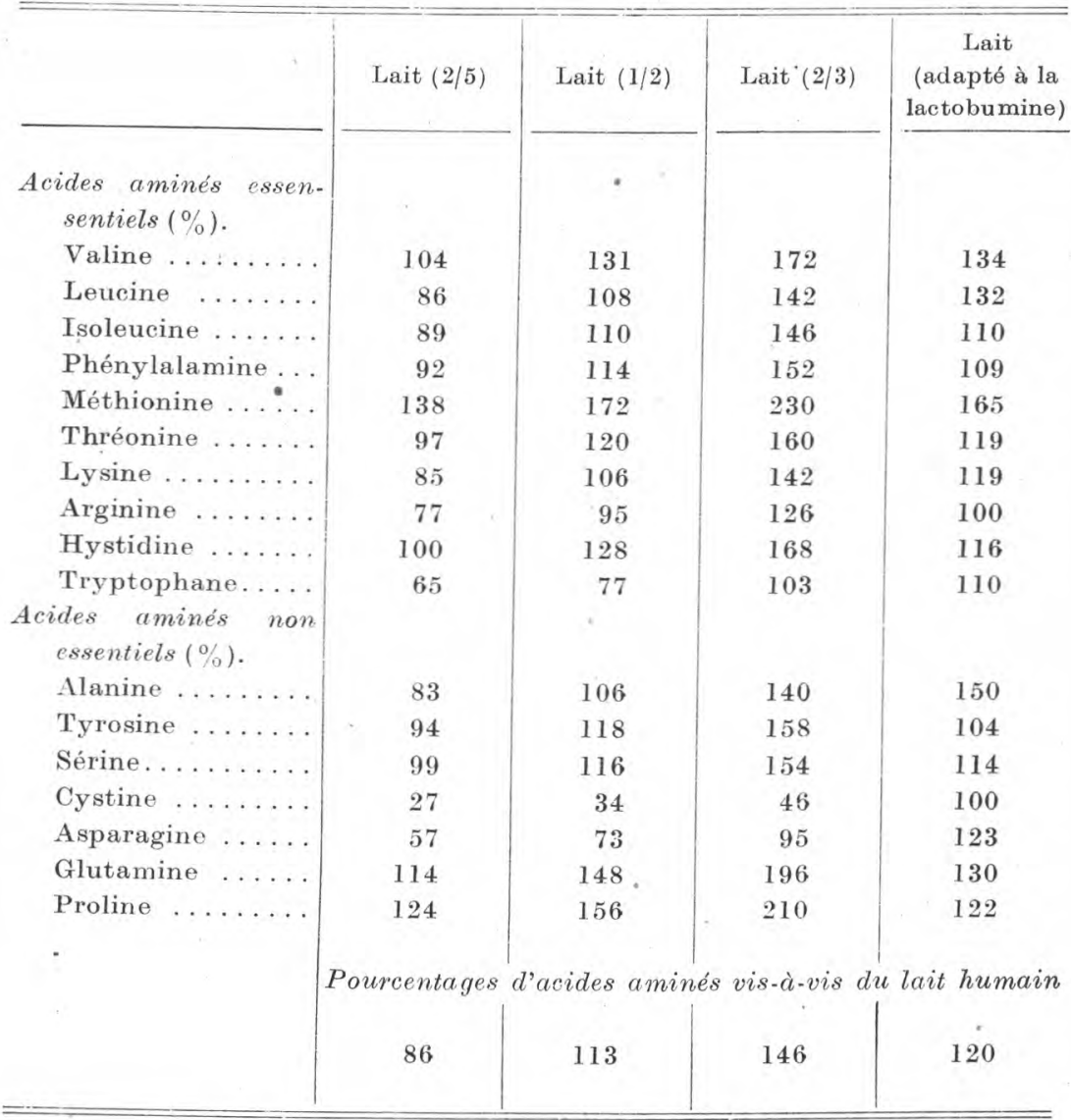

En raison de la teneur différente en sels des deux laits, une simple dilution, même si elle est égale à la majorité des sels, laisse les autres en excès ou en défaut.

Le coagulum du lait de vache est plus compact et se forme en 
masses plus grandes que dans le lait humain. Il demeure plus de temps dans l'estomac du nourrisson, étant plus difficilement attaquable par les sucs gastriques.

La caséine du lait de vache précipite en coagulats grands et durs, se différenciant en cela du coagulum fin et velouté du lait humain (BIEDERT).

La technique lactologique prépare actuellement des laits spéciaux (à partir de laits de vache), dont la composition chimique obéit au critérium d'obtenir des coagulums semblables à ceux du lait humain mais c'est un remède plus apparent que réel.

\section{Matières grasses}

La composition chimique de la matière grasse du lait de vache se différencie de celle du lait humain, en ce qu'elle accuse plus de tripalmitine et moins de trioléine.

Cette différence possède ųne importance pratique, car les savons de calcium et de magnésium de l'acide palmitique sont beaucoup moins facilement absorbés par le système intestinal que le sont ceux de l'acide oléique.

Le lait de vache contient aussi une proportion considérable de glycérides des acides plus « bas » ou volatils, qui irritent très souvent le système intestinal, provoquant des diarrhées. La considération du type de graisse comme fait indispensable dans la composition des laits maternisés est une découverte des dix dernières années.

C'est ce qui a été admirablement démontré par Frontali (Gli olî vegetali nell'alimentazione del lattante, 1938 ": les huiles végétales dans l'alimentation du nourrisson), par un tableau - que nous reproduisons en partie - donnant les différents pourcentages d'assimilation des substances grasses employées pour la préparation des aliments :

\begin{tabular}{|c|c|c|c|c|c|}
\hline Préparation lactée avec & $\begin{array}{c}\text { Indice } \\
\text { d'iode de } \\
\text { la matière } \\
\text { grasse }\end{array}$ & $\begin{array}{c}\text { Ingestion } \\
\%\end{array}$ & $\begin{array}{l}\text { Elimina. } \\
\text { tion par } \\
\text { les exeré- } \\
\text { ments \% }\end{array}$ & $\begin{array}{c}\text { Reste } \\
\%\end{array}$ & $\begin{array}{c}\text { Assimila- } \\
\text { tion } \%\end{array}$ \\
\hline Huile d'olive ....... & 83,4 & 11,90 & 1,48 & 10,42 & 87,16 \\
\hline Huile d'olive ....... & 83,4 & 14,19 & 1,27 & 12,92 & 91,05 \\
\hline Beurre $\{1) \ldots \ldots \ldots$ & 30,7 & 12,40 & 5,30 & 7,10 & 57,25 \\
\hline Beurre........... & 30,7 & 11,50 & 5,20 & 6,30 & 54,78 \\
\hline
\end{tabular}

et il conclut : «Les recherches expérimentales et cliniques sur des

(1) Vis-à-vis des variations possibles de qualité du beurre, l'huile d'olive est assurément plus stable. 
nourrissons normaux ou en conditions pathologiques caractérisées par une assimilation limitée des matières grasses, ont mis en évidence les taux d'assimilation optima de l'huile d'olive et des huiles végétales en général, en raison directe de leur plus grande teneur en trioléine, et donnant lieu à des développements immédiats, normaux et continus."

Ces formules, en MOYennes, Se COMposent AINSI :

\begin{tabular}{|c|c|}
\hline & Grammes \\
\hline Lait de vache $\ldots \ldots \ldots \ldots \ldots$ & 500 \\
\hline Eau $\ldots \ldots \ldots \ldots \ldots \ldots \ldots \ldots \ldots \ldots \ldots$ & 500 \\
\hline Sucre $\ldots \ldots \ldots \ldots \ldots \ldots \ldots \ldots \ldots \ldots \ldots$ & $30-50$ \\
\hline Farine de blé $\ldots \ldots \ldots \ldots \ldots \ldots \ldots \ldots \ldots \ldots$ & 40 \\
\hline Huile d'olive . . . . . . . . . . . . . . . . . & $30-50$ \\
\hline
\end{tabular}

\section{Sels}

Les sels sont nécessaires à la digestion et à toutes les étapes du métabolisme, depuis l'absorption jusqu'à la sécrétion et l'excrétion.

Le rôle que remplissent les sels, aussi bien dans les conditions normales que pathologiques, possède une importance augmentée par les études et recherches effectuées dans les dernières années (Rosell J. M. "Les connaissances actuelles sur le métabolisme minéral, 2 e édition, Barcelone, 1928).

Le lait humain contient 0 gr. 2 de sels minéraux dans $100 \mathrm{~cm}^{3}$, et le lait de vache $0 \mathrm{gr} .75$.

Tous les sels, sauf ceux qui contiennent du fer, se trouvent en plus grandes quantités dans le lait de vache que dans le lait humain. Le lait de vache contient, relativement, une très grande quantité de phosphate de calcium, tandis que la quantité de fer y est moins grande que dans le lait humain.

Le lait humain et le lait de vache diffèrent beaucoup par la forme du phosphore contenu. Dans le lait de femme, les trois quarts sont en combinaison organique, alors que dans le lait de vache, un quart seulement est combiné de cette manière.

$\mathrm{Ni}$ dans le lait humain, ni dans celui de vache, la teneur en fer n'est suffisante pour satisfaire les exigences de la première année de vie ; l'enfant doit dépendre du fer mis en réserve pendant sa vie fœtale.

Les pourcentages et poids en grammes des différents sels du lait humain et du lait de vache dans 100 parties de cendres, sont indiqués dans le tableau ci-après.

Dans tous les composants, à part le pentoxyde de phosphore et le fer, les pourcentages des différents sels sont virtuellement les mêmes dans les deux laits. La proportion plus grande de p’hosphore dans le lait de vache est due à la grande quantité de caséine. La 
SELS DU LAIT HUMAIN ET DU LAIT DE VACHE

Pocrcentages moyens des différents sels dans les Cendres

\begin{tabular}{|c|c|c|c|c|c|c|c|}
\hline & $\mathrm{CaO}$ & $\mathrm{MgO}$ & $\mathrm{P}^{2} \mathrm{O}^{5}$ & $\mathrm{Na}^{2} \mathrm{O}$ & $\mathrm{K}^{2} \mathrm{O}$ & $\mathrm{Cl}$ & $\mathrm{Fe}$ \\
\hline Lait humain. & 23,3 & 3,7 & 16,6 & 7,2 & 28,3 & 16,5 & 0,00015 \\
\hline Lait de vache. & 23,5 & 2,8 & 26,5 & 7,2 & 24,9 & 13,6 & 0,00007 \\
\hline
\end{tabular}

POIDS EN GRAMMES DE SELS PAR $100 \mathrm{CM}^{3}$ DE LAIT

\begin{tabular}{|c|c|c|c|c|c|c|c|}
\hline & $\mathrm{CaO}$ & $\mathrm{MgO}$ & $\mathrm{P}^{2} \mathrm{O}^{5}$ & $\mathrm{Na}^{2} \mathrm{O}$ & $\mathrm{K}^{2} \mathrm{O}$ & $\mathrm{NaCl}$ & $\mathrm{Fe}$ \\
\hline Lait humain. & 0,0458 & 0,0074 & 0,0345 & 0,0132 & 0,609 & 0,0358 & 0,00017 \\
\hline Lait de vache. & 0,172 & 0,02 & 0,2437 & 0,0465 & 0,1885 & 0,082 & 0,00007 \\
\hline
\end{tabular}

proportion de sels dans le lait de vache est presque la même que dans le lait humain, mais la quantité est triple. Par conséquent, si l'on dilue le lait de vache avec plus du double de son volume, ces éléments non organiques sont fournis à l'enfant en proportion égale à celle du lait humain. Celui-ci contient près du double de fer par rapport au lait de vache; de la dilution de ce dernier résulte une diminution excessive de la teneur en fer, si on ne la compense pas avec un autre aliment qui contient du fer.

Il est aussi naturel qu'idéologique que le lait de vache ait une composition minérale qualitative et quantitative différente de celle du lait de femme, et également que ses protéines, matières grasses et autres composants soient différents ; en effet, l'un est mathématiquement constitué pour former l'organisme du veau, l'autre, celui de l'enfant ou nourrisson humain. On sait que les cendres ou éléments minéraux du lait de femme sont, au milligramme près, exactement égales, qualitativement et quantitativement, aux cendres de l'organisme du fotus ou du nourrisson humain. De même pour le lait de vache ou d'autres mammifères vis-à-vis du veau ou autre jeune animal, "formés " intégralement par lesdits laits correspondants.

Au début, nous nous sommes référés aux études de Percival, Nilchelson, Finkelstein, Müller, Gonce, Marriot, Hesen, Masner, Kerley, Brenne, Mam, Bessau et autres, qui ont montré que les laits maternisés qui s'employaient, et dans une certaine mesure s'emploient encore, préparés sur la base de simples dilutions de lait de vache ou de chèvre, avec addition de lactose, farines lactées ou dextrines, étaient ou sont très loin de posséder les qualités digestives, les caractéristiques chimiques et particulièrement biologiques du lait maternel humain. 
Les nouvelles études et expériences, spécialement dans les cliniques pédiâtriques, pouponnières, etc,, qui se sont succédé sans interruption et donné naissance à une abondante bibliographie sur le sujet, ont montré, chaque fois avec plus de précision, que les laits maternisés obtenus par les méthodes de simples dilutions, addition de lactose, de farines dextrinées, présentent en autres les inconvénients suivants :

a) En parvenant à l'estomac du nourrisson, ils forment un coagulum dur et compact, difficilement digestible par les sucs gastriques, ce qui est dû à ce que la caséine du lait de vache ne forme pas un coagulum tendre, en flocons ou facilement désagrégeable comme celui du lait humain ;

b) Les matières grasses du lait de vache diffèrent au plus haut point de celles du lait humain par leur teneur en glycérides et en acides gras volatils, car la matière grasse du lait de vache ou de chèvre en contient jusqu'à dix fois plus que le lait de femme.

\section{Le type de matière grasse du lait humain et celui du lait de vache}

Dans les laits maternisés présentés ou recommandés jusqu'à des dates récentes, et si étrange que cela paraisse, on tenait compte seulement d'une approximation "grosso modo" de deux composants du lait maternel : caséine et lactose, ayant toujours négligé la valeur véritable des matières grasses, sels et lactoprotéines dans la composition des deux laits, comme le firent remarquer les pédiâtres connus Czerny et Keller. Ceux-ci mirent en lumière l'importance de modifier le caractère des matières grasses propres $\mathrm{du}$ lait de vache, si l'on prétend maintenir dans ces produits de substitution les propriétés biologiques et chimiques et la grande valeur nutritive des matières grasses du lait humain. Dans cette conception, ces auteurs ont considéré comme responsable des diarrhées infantiles et des vomissements, la présence d'une quantité d'acides gras et volatils quelque dix fois plus grande dans le lait de vache que dans le lait humain. L'examen des deux matières grasses, (de vache et humaine), qui possèdent des constantes physiques et chimiques différentes, l'a confirmé au moyen des chiffres suivants :

La matière grasse du lait de vache a un indice de réfraction de 1,4522-1,4580 contre 1,4568-1,4585 pour le lait maternel. Le chiffre de saponification est, respectivement, de 215-225 contre 205-209 ; l'indice de Reichert-Meissl est de 23-29 contre 1,50 à 21,10, et l'indice d'iode est de 23-29 en lait de vache contre $45,50-46,80$ en lait maternel. 
Pour cette raison, on commença d'importants essais, et depuis lors, les projets furent nombreux pour la préparation de laits à matière grasse humanisée, si l'on peut s'exprimer ainsi.

En 1912, le chimiste allemand Arnold proposait l'emploi du beurre de coco et du saindoux de porc, afin que les constantes physiques et chimiques coincident le plus possible avec celles des matières grasses du lait maternel. Le pédiâtre GERstenberGer (Etats-Unis) utilisa, depuis 1919, une matière grasse qui approche de très près les constantes désirées, obtenue par un mélange de matières grasses (de vache, huile de coco, beurre, beurre de cacao et huile de foie de morue), employant l'huile de foie de morue pour conférer au lait un pouvoir antirachitique et provoquer la bonne régénération calcique de l'enfant.

Les matières minérales diffèrent aussi, en particulier quantitativement, de celles du lait humain, qui en contient un peu moins de la moitié de la teneur du lait de vache, parce que le lait de cet animal doit former en moins de temps un volume plus grand de squelette que le lait humain n'a à former pour l'enfant. Les lactoprotéines existent au contraire en plus petite quantité dans le lait de vache que dans le lait humain; en ce qui concerne le lactose et la caséine, seules différences qu'on avait l'habitude de prendre en considération jusqu'à présent pour les laits usuels dénommés maternisés, qu'ils soient liquides, condensés ou en poudre, la diversité entre les deux laits est bien connue, comme il a été exposé précédemment.

Nous savons que la composition correcte de l'aliment artificiel doit comprendre tous les principes immédiats du lait de femme, et dans la même proportion que les contient celui-ci. La proportion entre les albumines, les graisses et les hydrates de carbone doit être :

$$
1: 3,5: 7
$$

et, dans cette proportion optima elle-même, le rapport des matières grasses et des hydrates de carbone doit être de :

$$
1: 2 \text {. }
$$

On doit toujours tenir compte de la relation intime entre le métabolisme des matières grasses et des hydrates de carbone, pour "refaire" un aliment, puisque, pour une quantité déterminée de matières grasses, il doit renfermer le double d'hydrates de carbone.

A l'instar du calcium et du potassium dont les rôles dans le métabolisme minéral sont antagonistes, les matières grasses et les hydrates de carbone ont, eux aussi, une fonction antagoniste, comme l'on en juge par ce qui suit :

Les matières grasses :

- Retardent le métabolisme ; 
- Ont une oxydation lente ;

- S'opposent à la rétention de l'eau ;

- Procurent une augmentation de poids stable;

- Augmentent l'immunité.

Les hydrates de carbone:

- Accélèrent le métabolisme ;

- Sont d'oxydation rapide ;

- Favorisent la rétention de l'eau;

- L'augmentation de poids est instable ;

- Donnés en excès, ils diminuent l'immunité.

Pour ce qui a trait à la compensation de leurs proportions respectives, on parviendra à les insérer dans l'aliment suivant les normes précitées.

Et puisque nous faisons mention des rapports, nous voyons aussi que le rapport de la caséine à l'albumine est de $6: 1$, et dans le lait de femme, de $4: 1$; c'est pourquoi, si l'on veut rapprocher le lait de vache du lait humain par dilution avec de l'eau et addition d'hydrates de carbone, on ne doit pas omettre d'ajouter l'albumine, ce qui a déjà été essayé, spécialement en Amérique.

Il est évident que si les corrélations et les normes quantitatives ont de nos jours une importance, les travaux modernes mettent au premier plan cette importance considérable de la valeur quantitative des différents composants du lait.

Avant d'exposer l'étude des bases chimiques et biologiques du type de lait qui, jusqu'à aujourd'hui, peut être considéré comme le plus parfait qui existe, sur le terrain des laits humains ou humanisés, nous croyons convenable d'exposer quelques données des études évolutives qui ont été faites ces derniers temps.

Pour reproduire quelques-unes des opinions émises sur les laits " modifiés humanisés ", nous résumons certains extraits des plus récentes œuvres de pédiâtres, entre autres, celle de Presick : «Un aliment complet se compose de beaucoup de constituąnts divers. »

Depuis un temps immémorial, on a élaboré des produits de substitution du lait de femme avec des aliments qui, selon les connaissances de l'époque, étaient supposés posséder des propriétés similaires à celles de l'aliment naturel. Cependant, la connaissance incomplète du modèle explique pourquoi la majorité desdits aliments lui sont si différents, en qualité et dans leurs résultats. En effet, le lait contient environ quarante composants distincts, y compris six vitamines différentes et plus d'une douzaine de matières minérales diverses, outre les variétés de matières grasses et acides gras, différentes protéines et extraits aromatiques et colorants, 
dont la majorité possède un rôle dans la nutrition. Connaissant cette complexité du lait humain, il se conçoit aisément que quiconque essaie d'élaborer un produit de remplacement s'expose à des omissions ou à des erreurs.

Chacun des nombreux composants du lait jouit d'un pouvoir nutritif distinct, chacun d'eux joue un rôle certain dans le maintien de la santé; on ne peut en faire abstraction pour obtenir les meilleurs résultats. Si l'on oublie complètement l'un quelconque des pripcipaux constituants, de graves conséquences peuvent survenir plus ou moins rapidement; si on l'administre dans une proportion très lointaine de la proportion optima, la nutrition peut en supporter les effets avec une rapidité plus ou moins grande.

Les meilleurs résultats s'obtiennent seulement si l'on administre tous les composants cités dans une proportion optima. Si un facteur alimentaire aussi important que, par exemple, la vitamine $\mathrm{C}$ ou antiscorbutique, manque complètement dans l'alimentation du nourrisson, la conséquence fatale en est la mort par scorbut (c'està-dire une maladie qui se manifestera avec ses symptômes caractéristiques) au bout de trois mois environ, parfois avant. Si l'alimentation contient ladite vitamine, mais en quantité inférieure à l'optimum précisé, l'enfant ne meurt pas, mais le stade de nutrition est inférieur à celui qui existerait si l'aliment avait été administré en la quantité prescrite.

De semblables résultats sont la suite de la carence totale ou d'un déficit partiel de la majorité des principaux constituants de l'alimentation; mais jusqu'à présent, en raison de nos connaissances limitées sur le résultat des carences et leurs méthodes d'examen, il n'est pas toujours possible d'attribuer à leur véritable cause les symptômes observés en maintes circonstances. Assurément, nous avons une certaine connaissance en ce qui concerne les manifestations cliniques auxquelles il faut s'attendre dans les cas, non compliqués, de déficience vitaminée $\mathrm{A}, \mathrm{B}, \mathrm{C}$ et $\mathrm{D}$, ainsi que des déficits en fer, iode, calcium, phosphore et chlorure de sodium. Mais il est habituellement difficile d'interpréter les résultats d'un déficit mixte de plusieurs desdits composants. Nous mentionnons ces faits fondamentaux dans le seul but de signaler qu'une nutrition parfaite ne dépend pas seulement d'un apport optimum de quelques substances alimentaires, telles que l'albumine, les hydrates de carbone et les matières grasses, mais de beaucoup d'autres choses; et d'autre part que toutes ces substances ne sont pas seulement présentes dans le lait humain, mais qu'elles le sont dans la proportion indispensable et optima.

Dans la plupart des régimes, il manque des éléments essentiels. Les résultats, peu satisfaisants, de l'état nutritif de la population 
adulte de presque tous les pays du monde se doivent, en grande partie, à des défauts du régime, qui ne fournit pas tous les éléments essentiels pour la nutrition.

En conséquence, nous pouvons résumer le principe fondamental de l'alimentation physiologique dans les termes suivants :

Pour le maintien d'un état nutritif parfait, il est exigé un grand nombre de substances alimentaires diverses, $y$ compris vitamines et sels (une quarantaine au total); et pour obtenir cet état nutritif optimum, il faut les administrer en quantités optimum.

Nous devons nous souvenir qu'outre les prineipes immédiats, une autre série de composants de l'aliment est indispensable à la nutrition; par exemple, il y a lieu d'apporter cinq sortes de vitamines et au moins une douzaine de sels, chacun d'eux jouant un rôle important dans divers processus du métabolisme, dont dépendent la santé et la croissance. Doit se considérer comme optima la proportion dans laquelle ces divers facteurs accessoires se trouvent présents dans le lait de femme, en quantités si minimes qu'il est évidemment difficile de les administrer en égales proportions dans les aliments artificiels. Par exemple, il est très important d'administrer à l'enfant une quantité adéquate de fer, d'iode, de vitamine D, aussi bien s'il est allaité au sein que s'il est nourri au biberon; mais la quantité des facteurs alimentaires mentionnés varie avec les différents types de laits humains, et parfois, elle se trouve endessous du minimum nécessaire; ce même danger est encore plus grand avec un aliment artificiel, non seulement parce que la proportion est habituellement inférieure à celle du lait de femme, mais par le fait de la dilution que l'on fait subir au lait de vache pour le rendre apte à l'alimentation du nourrisson.

Par conséquent, dans la modification du lait de vache, il ne suffit pas de se borner à obtenir ladite similitude en ce qui concerne les albuminoïdes, hydrates de carbone et matières grasses, mais il faut aussi considérer le pourcentage de ces aliments essentiels, dans la mesure où la pratique permet de les administrer dans les quantités exactes requises.

\section{Inconvénients de quelques-unes des méthodes employées actuellement pour modifier le lait de vache destiné au nourrisson}

\section{Lait à la moitié et au tiers.}

Il consiste à diluer le lait avec une quantité égale ou de deux tiers d'eau, en additionnant de la crème et du sucre pour élever le pourcentage de matières grasses et d'hydrates de carbone à la mesure idéale du lait de femme. Les détails sont les suivants : 


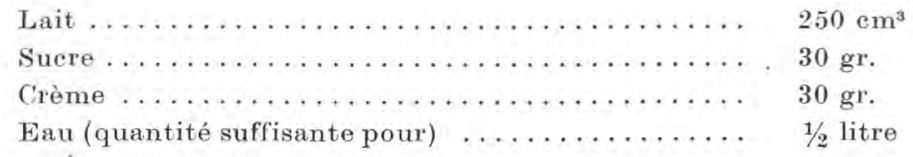

Avantages : simplicité de préparation.

\section{Inconvénients.}

Cette méthode n'essaie pas de corriger la proportion incorrecte du lait de vache entre albuminoïdes solubles et insolubles (caséine). Le total des protéines y est légèrement supérieur à celui du lait humain, et elles sont de qualité plus indigeste. En comparaison avec le lait humain, il y a déficit en vitamines $\mathrm{B} 1, \mathrm{~B} 2$ et $\mathrm{C}$, en tous les sels (à l'exception du phosphate calcique) et en substances extractives, y compris lécithine et cholestérine. Une partie de ces défauts peuvent se corriger en remplaçant les $100 \mathrm{~cm}^{3}$ d'eau par la même quantité de bouillon d'os et de légumes.

\section{Méthode de modification du lait de vache par réduction de la caséine}

Cette méthode essaie de corriger le rapport défectueux du lait de vache (et dans ses dilutions habituelles) entre la caséine et les autres protéines, dans le but de les ajuster à leur proportion dans le lait humain.

Dans le lait de vache, le pourcentage approximatif de caséine est de $3 \%$, tandis que les autres protéines (parmi lesquelles la lactoalbumine et la lactoglobuline) sont présentes à raison de moins de $1 \%$. Dans le lait humain, la proportion est de $0,5 \%$ de caséine et $1 \%$ de séroalbumine; en d'autres termes, le lait de vache a six fois plus de caséine que celui de femme, tandis que la teneur en séroalbumine est de la moitié environ.

Cette discordance entre les deux sortes de lait détermine fréquemment des réactions pathologiques, de caractère parfois grave, qui affectent l'enfant lorsqu'il passe du lait maternel au lait de vache.

En premier lieu, la caséine du lait de vache est beaucoup plus difficile à digérer que la séro-albumine, principalement parce qu'elle précipite dans l'estomac en un coagulum pesant, "paresseusement " soluble, qui doit se dissoudre pour pouvoir passer au duodénum; et en second lieu, à cause de son grand pouvoir d'obstacle sur l'acide chlorhydrique sécrété par la muqueuse gastrique. En conséquence, pour que le $p \mathrm{H}$ du chyme atteigne le point nécessaire pour activer la pepsine et digérer la caséine, une grande quantité d'acide doit être sécrétée. Cela signifie que si toute la caséine se 
digère complètement, les glandes gastriques développent à l'excès leur fonction oxynthique rendant très probable la production d'un état permanent d'hyperchlorhydrie.

De temps à autre, diverses méthodes ont été proposées pour obvier à cette disproportion, diminuant à la fois la quantité de caséine sans abaisser les séroalbumines au-dessous des exigences physiologiques. En effet, les différences de la teneur en acides aminés de la caséine et des lactoalbumines, ainsi que la diversité qui en résulte quant à leur valeur biologique, explique le fait que l'on ne peut compenser le déficit des premières par un excès des secondes, même en les administrant en grand excès.

Il s'avère relativement facile de séparer toute la caséine du lait, en la coagulant au moyen de présure et en filtrant ensuite ; mais il est difficile. d'en enlever seulement une partie pour ne laisser dans le filtre que $0,5 \%$. Cette difficulté peut se vaincre en additionnant au produit filtré une partie du coagulum de caséine jusqu'à obtention des $0,5 \%$ désirés. A cet égard, on se doit de considérer que la caséine n'étant pas à l'état liquide, mais solide, difficilement mélangeable dans le produit filtré, on obtiendra ce mélange en agitant avec une partie de ce produit filtré puis en ajoutant le reste.

\section{A. Lait avec acide lactique et avec acide citrique}

Quand on ajoute de l'acide lactique au lait, la caséine précipite ; le même résultat-se produit par addition d'acide citrique, de jus de citron ou d'acide chlorhydrique en quantités adéquates, ou lorsque les bactéries lactiques se multiplient dans le lait. Si le coagulum ainsi formé se délaye, par agitation ou action similaire, le produit qui en résulte constitue un aliment plus précieux pour les nourrissons malades ou fragiles.

\section{Avantages}

Comme la caséine a été coagulée, et le coagulum divisé en fins flocons, elle se présente très divisée à l'action du suc gastrique, et pour cette raison, sous la forme la plus favorable à sa " peptonisation ". D'autre part, sa réaction acide garantit un $p \mathrm{H}$ adéquat pour la meilleure digestion des protéines, anihilant la propriété "tampon " (obstacle) du lait de vache ordinaire. On doit utiliser un lait adapté quant à sa teneur en caséine.

\section{Lait « lactoacidifié ».}

Ce terme doit être réservé au lait contenant l'acide lactique produit par la fermentation naturelle du lactose qu'il contient, sous l'influence des microorganismes de la fermentation lactique, qu'il 
s'agisse du B. bulgaricus, du B. acidophilus, du Streptococcus lactis, ou autres.

\section{Avantages.}

Le lait acide ainsi préparé a les avantages du lait additionné d'acide lactique, et en outre, celui provenant de ce que les germes d'acidité continuent à se multiplier dans l'intestin, favorisant la prolifération de la flore saccharolytique et faisant obstacle à la flore protéolytique défavorable.

Les excréments du nourrisson alimenté quelque temps avec du "lait acide lactique" doivent être inodores, ce qui prouve l'action neutralisante des germes de la fermentation lactique sur les microbes de la putréfaction, causes de la décomposition de la caséine.

Le lait doit également être adapté quant aux proportions de protéines, matières grasses et autres éléments.

\section{Lait citraté.}

Obtenu en incorporant au lait des. quantités dissemblables de eitrate sodique, s'appliquant aussi bien au lait pur qu'à ses dilutions. L'incorporation de citrate a pour objet de transformer le caséinate de calcium en caséinate sodique, lequel, sous l'influence du «lab» (présure) de la pepsine du suc gastrique, se convertit en paracaséinate sodique, qui, à l'encontre du paracaséinate calcique, est soluble dans l'eau,

Dans les laits humanisés considérés comme les plus parfaits, la modification de la caséine du lait de vache (pour lui donner une constitution de type physique similaire au lait humain) s'obtient par l'emploi mixte de l'acide citrique ou jus de citron et du citrate sodique, - très employé dans la plupart des cliniques pédiatriques d'Allemagne, des Etats-Unis et d'autres pays, - avec celui des bactéries acidoprotéolytiques, de façon que l'action produite soit en quelque sorte une pré-digestion partielle de la caséine. Par ce procédé mixte on atteint, en même temps, le double but d'apporter au lait d'une part, une plus grande quantité de vitamines $C$ (jus de citron), d'autre part, une quantité appréciable de vitamine B2 ou lactoflavine (ou riboflavine) que produisent lesdites bactéries dans le sérum du lait. L'usage des décoctions de çéréales ou de légumes en proportions bien définies comme liquide de dilution contribue à la floculation plus fine de la caséine et apportent les trois types - ou plus - d'hydrates de carbone, de vitamines de certains légumes (carotène et sels de fer), dans ce type de lait qu'il y a lieu de considérer aujourd'hui comme le plus approchant de l'idéal.

Le docteur EscuDERo a utilisé cette formule avec de très bons résultats : 


\section{Grammes}

Lait de vache . . . . . . . . . . . . . . . . . 500

Mucilage d'orge ................... 376

Sucre ordinaire $\ldots \ldots \ldots \ldots \ldots \ldots \ldots \ldots \ldots \ldots \ldots \ldots$

Crème homogénéisée à $40 \%$ de matière grasse butyro-

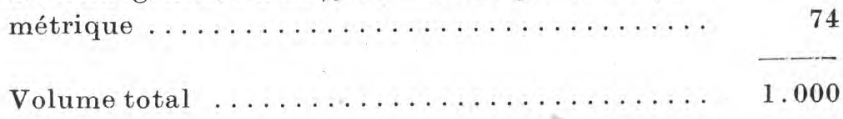

Ce lait modifié, comparé au lait humain, accuse les résultats analytiques ci-après :

Références par $100 \mathrm{ml}$.

1. Valeur calorique totale

2. Pourcentage :

Hydrates de carbone ..........
Protéines .............

Matières grasses ............

3. Quotients :

Calorie-gramme ...........

Cétoanticétogène ............

Calcium-phosphore .........

Potassium-sodium ..........

Potassium-calcium .........

Unités basiques. . . . . . . . . . .
Lait humain

71 calories

$40,1 \%$

$6,4 \%$

$53,4 \%$

1,40

0,54

2

1,75

1,92

1
Mélange lacté

71 calories

$38,9 \%$

$7,9 \%$

$53,2 \%$

1,40

0,54

1,15

4,31

1,34

0,59

\section{TABLEAU GOMPARATIF DE LA FORMULE DÉVLLPPÉE DU LAIT HUMAIN ET} DU MÉLANGE LAGTÉ ESGUDERO

\section{VALEUR CALORIQUE ET PLASTIQUE}

Références par $100 \mathrm{ml}$. Lait humain “entier" Mélange lacté (grammes par $100 \mathrm{ml}$.)

\begin{tabular}{|c|c|c|c|c|}
\hline Hydrates de carbone ......... & \multicolumn{2}{|l|}{7,13} & \multicolumn{2}{|l|}{6,92} \\
\hline Protéines $\ldots \ldots \ldots \ldots \ldots \ldots$ & \multicolumn{2}{|l|}{1,14} & \multicolumn{2}{|l|}{1,40} \\
\hline Matières grasses $\ldots \ldots \ldots \ldots \ldots$ & \multicolumn{2}{|c|}{4,22} & \multicolumn{2}{|l|}{4,20} \\
\hline Cendres totales $\ldots \ldots \ldots \ldots \ldots$ & \multicolumn{2}{|c|}{0,218} & \multicolumn{2}{|l|}{0,24} \\
\hline Calcium $\ldots \ldots \ldots \ldots \ldots \ldots$ & 26 & $\mathrm{mg}$. & 49 & $\mathrm{mg}$. \\
\hline Phosphore $\ldots \ldots \ldots \ldots \ldots \ldots$ & 13,8 & " & 43 & 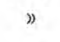 \\
\hline Chlore .................. & 32,5 & ” & 40,5 & 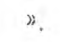 \\
\hline Sodium $\ldots \ldots \ldots \ldots \ldots \ldots$ & 28,5 & j & 15 & ) \\
\hline Potassium $\ldots \ldots \ldots \ldots \ldots \ldots$ & 50 & 》 & 66 & $»$. \\
\hline Soufre ................. & 8 & » & - & ” \\
\hline Magnésium ................ & 6 & $\eta$ & $\longrightarrow$ & ) \\
\hline Fer $\ldots \ldots \ldots \ldots \ldots \ldots \ldots$ & 0,06 & $n$ & 0,14 & " \\
\hline Cuivre .............. & 0,03 & $"$ & 0,008 & $n$ \\
\hline
\end{tabular}

VALEUR EN VITAMINES

\begin{tabular}{|c|c|c|}
\hline Vitamine $A \ldots \ldots \ldots \ldots$ & $308 \quad$ U.I. & $524 \quad$ U.I. \\
\hline Vitamine D & 0,83 U.I. & 9,05 U.I. \\
\hline
\end{tabular}




\begin{tabular}{|c|c|c|c|}
\hline$\ldots \ldots \ldots \ldots \ldots$ & 57 & gammas & 29 \\
\hline Vitamine $\mathrm{B} 2 \quad \ldots \ldots \ldots \ldots \ldots$ & 20 & gammas & 89 \\
\hline Acide nicotinique $\ldots . \ldots \ldots \ldots$ & traces & & 93 \\
\hline Vitamine $\mathrm{C} \ldots \ldots \ldots \ldots \ldots \ldots$ & 4,1 & $\mathrm{mg}$. & 0,5 \\
\hline
\end{tabular}

\section{B. Aspect du type de matière grasse}

Pour l'adaptation des matières grasses et pour parvenir à ce que le lait humanisé ait un type de matière grasse le plus semblable possible, par sa constitution, au lait maternel, on remplacera une partie de la crème du lait de vache par une proportion convenable d'huile d'olive, de soja, et éventuellement de coco. Par cette combinaison d'une partie de la crème de lait avec d'autres, correspondant aux huiles plus digestibles mentionnées, on obtiendra en outre un type de graisse de conditions biologiques (en ce qui concerne les glycérides) beaucoup plus ressemblantes, presque similaires à la matière grasse du lait humain. Par un chauffage adéquat de la matière grasse du lait de vache, on peut libérer celle-ci de l'excès d'acides gras.

Nous poursuivons en exposant la composition en glycérides du lait de vache, du lait maternel et du lait humanisé qu'il y a lieu de considérer aujourd'hui comme le plus parfait des produits de remplacement du lait maternel. Ceux-ci parviennent difficilement au stade de succédanés physiologiquement parfaits du lait maternel, bien que sous certains aspects ils peuvent lui être supérieurs; par exemple, par leur teneur plus constante, et par leur contenu intensifié de vitamines et de fer, souvent déficitaires dans le lait humain.

\begin{tabular}{|c|c|c|c|}
\hline & $\begin{array}{c}\text { Matière grasse } \\
\text { du lait de vache } \\
\%\end{array}$ & $\begin{array}{c}\text { Matière grasse } \\
\text { du lait maternel } \\
\%\end{array}$ & $\begin{array}{c}\text { Matière grasse } \\
\text { du lait humanisé } \\
\text { (type amélioré) } \\
\%\end{array}$ \\
\hline Stéarine $\ldots \ldots \ldots$. & 2 & 5 & 4 \\
\hline Palmitine.......... & 35 & 19 & 17 \\
\hline Oléine............. & 32 & 76 & 77 \\
\hline
\end{tabular}

\section{Adaptation de la lactoprotéine}

Par les dilutions courantes des biberons, en ajoutant de l'eau pour diluer la caséine dans la proportion convenable, on dilue aussi, en même temps que les matières grasses et le lactose, les lactoprotéines ou lactoalbumines qui existent déjà en quantité inférieure dans le lait de vache, par rapport au lait humain.

$\mathrm{Si}$, par lesdites dilutions courantes, on diminue à moins du tiers la caséine du lait de vache (dont la proportion approximative est de $3 \%$ dans le lait de vache contre $0,75 \%$ dans le lait humain), on diminue en proportion égale la lactoalbumine, qui existe dans le 
lait de vache à raison de $0,33 \%$ environ contre $1,33 \%$ dans le lait humain. C'est pourquoi il est absolument nécessaire de tenir compte de ce fait, dans la préparation de biberons de laits maternisés, pour. qu'ils soient à la mesure des exigences actuelles.

Dans les dilutions courantes, les lactoprotéines diminuent dans une proportion considérable et anormale; et ceci au point qu'un lait dilué avec deux parties d'eau, au lieu de renfermer 1,33\% de lactoprotéines comme le lait humain, est abaissé à efviron 0,17 , et ceei avec un préjudice notable pour la nutrition de l'enfant; les lactoprotéines se réintègrent, dans les types de lait modernes, dans la proportion convenable obtenue du sérum du lait après précipitation de la caséine.

Et enfin, sur l'amélioration de l'emploi de ces laits biologiquement adaptés en partant du lait de vache, citons l'opinion de Finkelstìin : "Actuellement, en fin de compte, le lait de vache bien adapté se digère aussi bien que le lait maternel. "

Les différences qui existent, surtout la structure distincte des excreta, sont la conséquence naturelle de la composition propre de chaque constituant de l'aliment, qui exige un processus chimique digestif distinct et laisse des résidus différents; mais elles n'indiquent en aucune façon que ce processus digestif soit incomplet, dans les proportions choisies.

Selon les recherches sur le métabolisme du lait de vache bien préparé, l'absorption est, au contraire, excellente. La proportion de matière grasse dans les excreta ne représente que 3 à $4 \%$ de la matière grasse contenue dans l'aliment, et la proportion d'azote ne dépasse pas $5 \%$, dont la majeure partie ne provient pas des résidus alimentaires, mais des sécrétions intestinales et des bactéries de l'intestin.

En résumé, un lait destiné à l'allaitement artificiel ou mixte, et pouvant se dénommer "humanisé ", doit se préparer avec l'idée fondamentale de tenir compte : des différences de proportions de caséine, lactoprotéine, lactose, sels, entre le lait de vache et le lait humain; de la variété des composants des matières grasses de ces deux laits en glycérides et acides gras; de la différence de consistance du coagulum de la caséine des deux laits, qui doit être modifiée par les méthodes que nous enseigne la lactologie moderne.

Quant à la "vitaminisation " du lait en biberons stérilisés, on a obtenu actuellement des résultats qui peuvent se qualifier de parfaits, particulièrement fondés sur l'intervention de levures et germes de soja, qui contiennent toutes les vitamines requises et autres facteurs biologiques en quantité suffisante.

L'homogénéisation du lait de vache entre dans la pratique de 
l'amélioration du lait pour l'alimentation infantile (en réalité, les globules de matière grasse en suspension dans le lait sont divisés et subdivisés $5,7,10$ fois..., améliorant physiquement l'aliment, en le rendant plus digestif et plus nutritif).

Les laits destinés à l'alimentation artificielle ou non spécifique du nourrisson doivent être supportés de façon satisfaisante. Pour cela, la température, le nombre journalier et la quantité des doses seront fonction des désirs de l'enfant, des modalités de l'allaitement naturel et des indications du pédiatre.

Personne ne peut avoir la prétention de remplacer l'alimentation spécifique de l'enfant (étant admis que la donneuse soit saine et le lait complet et correspondant), par un lait de vache modifié ; mais on peut le rapprocher chimiquement et l'adapter physiologiquement pour qu'il remplisse la fonction du succédané.

Si avec un lait modifié déterminé, on obtient un développement normal de l'enfant, le maintien de la santé et une bonne immunité, le résultat sera satisfaisant.

\title{
Analyse des laits maternisés
}

Elle est basée sur les mêmes règles que celles qui ont été mentionnées pour le lait normal et le lait en poudre.

\section{LES FACTEURS BACTÉRIOLYTIQUES ANTITUBERCULEUX DES FROMAGES (1)}

\author{
par
}

J. MEYER, J. TOUILLIER et J. MALGRAS

Les bacilles tuberculeux du type bovin introduits dans les fromages par le lait tuberculeux se maintiennent vivants et virulents dans les fromages mous jusqu'au stade de la décomposition putride finale, alors que ces germes disparaissent par un phénomène d'autostérilisation au bout dos quatre premières semaines dans les fromages à pâte dure.

Or, on pourrait incriminer trois facteurs dans ce processus :

$1^{\circ}$ Le lait étant chauffé dans les récipients de cuivre, les traces de ce métal entrant en contact avec le caillé pourraient être rendues responsables de la lyse des bacilles tuberculeux.

$2^{\circ}$ La fermentation lactique et les lactates jouent-ils leur rôle dans ce processus?

$3^{\circ}$ Les germes de la fermentation propionique existant dans la préparation des fromages cuits seraient-ils les agents de la destruction des bacilles tuberculeux ?

(1) C. R. Acad. Agric., 1952, 4, 167. 